\title{
Efficient Privacy-Preserving Face Recognition
}

\author{
Ahmad-Reza Sadeghi, Thomas Schneider, and Immo Wehrenberg \\ Horst Görtz Institute for IT-Security, Ruhr-University Bochum, Germany \\ \{ahmad.sadeghi, thomas.schneider\}@trust.rub.de ${ }^{\star}$, immo.wehrenberg@rub.de
}

\begin{abstract}
Automatic recognition of human faces is becoming increasingly popular in civilian and law enforcement applications that require reliable recognition of humans. However, the rapid improvement and widespread deployment of this technology raises strong concerns regarding the violation of individuals' privacy. A typical application scenario for privacy-preserving face recognition concerns a client who privately searches for a specific face image in the face image database of a server.

In this paper we present a privacy-preserving face recognition scheme that substantially improves over previous work in terms of communicationand computation efficiency: the most recent proposal of Erkin et al. (PETS'09) requires $\mathcal{O}(\log M)$ rounds and computationally expensive operations on homomorphically encrypted data to recognize a face in a database of $M$ faces. Our improved scheme requires only $\mathcal{O}(1)$ rounds and has a substantially smaller online communication complexity (by a factor of 15 for each database entry) and less computation complexity.

Our solution is based on known cryptographic building blocks combining homomorphic encryption with garbled circuits. Our implementation results show the practicality of our scheme also for large databases (e.g., for $M=1000$ we need less than 13 seconds and less than 4 MByte online communication on two $2.4 \mathrm{GHz}$ PCs connected via Gigabit Ethernet).
\end{abstract}

Keywords: Secure Two-Party Computation, Face Recognition, Privacy.

\section{Introduction}

In the last decade biometric identification and authentication have increasingly gained importance for a variety of enterprise, civilian and law enforcement applications. Examples vary from fingerprinting and iris scanning systems, to voice and face recognition systems, etc. Many governments have already rolled out electronic passports [18] and IDs [27] that contain biometric information (e.g., image, fingerprints, and iris scan) of their legitimate holders.

In particular it seems that facial recognition systems have become popular aimed to be installed in surveillance of public places [17], and access and border control at airports 8 to name some. For some of these use cases one requires online search with short response times and low amount of online communication.

Moreover, face recognition is ubiquitously used also in online photo albums such as Google Picasa and social networking platforms such as Facebook which

\footnotetext{
^ Supported by EU FP6 project SPEED, EU FP7 project CACE and ECRYPT II.
} 
have become popular to share photos with family and friends. These platforms support automatic detection and tagging of faces in uploaded images 1 Additionally, images can be tagged with the place they were taken 2

The widespread use of such face recognition systems, however, raises also privacy risks since biometric information can be collected and misused to profile and track individuals against their will. These issues raise the desire to construct privacy-preserving face recognition systems 12 .

In this paper we concentrate on efficient privacy-preserving face recognition systems. The typical scenario here is a client-server application where the client needs to know whether a specific face image is contained in the database of a server with the following requirements: the client trusts the server to correctly perform the matching algorithm for the face recognition but without revealing any useful information to the server about the requested image as well as about the outcome of the matching algorithm. The server requires privacy of its database beyond the outcome of the matching algorithm to the client.

In the most recent proposal for privacy-preserving face recognition 12 the authors use the standard and popular Eigenface 3433 recognition algorithm and design a protocol that performs operations on encrypted images by means of homomorphic encryption schemes, more concretely, Pailler 2911 as well as a cryptographic protocol for comparing two Pailler-encrypted values based on the Damgård, Geisler and Krøigård 910] cryptosystem). They demonstrate that privacy-preserving face recognition is possible in principle and give required choices of parameter sizes to achieve a good classification rate. However, the proposed protocol requires $\mathcal{O}(\log N)$ rounds of online communication as well as computationally expensive operations on homomorphically encrypted data to recognize a face in the database of $N$ faces. Due to these restrictions, the proposed protocol cannot be deployed in practical large-scale applications. In this paper we address this aspect and show that one can do better w.r.t. efficiency.

Basically one can identify two approaches for secure computation: the first approach is to perform the required operations on encrypted data by means of homomorphic encryption (see, e.g., 2911]). The other approach is based on Garbled Circuit (GC) à la Yao [3522]: the function to be computed is represented by a garbled circuit i.e., the inputs and the function are encrypted ("garbled"). Then the client obliviously obtains the keys corresponding to his inputs and decrypts the garbled function. Homomorphic Encryption requires low communication complexity but huge round and computation complexity whereas GC has low online complexity (rounds, communication and computation) but large offline communication complexity. We present a protocol for privacy-preserving face recognition based on a hybrid protocol which combines the advantages of both approaches. A protocol based on GC only is given in the full version 32 .

\footnotetext{
${ }^{1}$ http://picasa.google.com/features-nametags.html; http://face.com

${ }^{2}$ Geotagging can be done either manually or automatically on iPhones using GPS http://www.saltpepper.net/geotag
} 
Contribution. We give an efficient and secure privacy-preserving face recognition protocol based on the Eigenfaces recognition algorithm 34/33 and a combination of known cryptographic techniques, in particular Homomorphic Encryption and Garbled Circuits. Our protocol substantially improves over previous work [12] as it has only a constant number of $\mathcal{O}(1)$ rounds and allows to shift most of the computation and communication into a pre-computation phase. The remaining online phase is highly efficient and allows for a quick response time which is especially important in applications such as biometric access control.

Related Work. Privacy-Preserving Face Recognition allows a client to obliviously detect if the image of a face is contained in a database of faces held by server. We give a detailed summary of previous work on privacy-preserving face recognition 12 in \$3.1. Our protocol has a substantially improved efficiency.

The related problem of Privacy-Preserving Face Detection [3] allows a client to detect faces on his image using a private classifier held by server without revealing the face or the classifier to the other party.

In order to preserve privacy, faces can be de-identified such that face recognition software cannot reliably recognize de-identified faces, even though many facial details are preserved as described in 28.

\section{Preliminaries}

In this section we summarize our conventions and setting in 2.1 and cryptographic tools used in our constructions in $\$ 2.2$ (additively homomorphic encryption (HE), oblivious transfer (OT), and garbled circuits (GC) with free XOR). A summary of the face recognition algorithm using Eigenfaces is given in 2.3 . Readers familiar with the prerequisites may safely skip to 3 .

\subsection{Parameters, Notation and Model}

We denote symmetric security parameter by $t$ and the asymmetric security parameter, i.e., bitlength of RSA moduli, by $T$. Recommended parameters for short-term security (until 2010) are for example $t=80$ and $T=1024$, whereas for long-term security $t=128$ and $T=3072$ are recommended [16. The statistical correctness parameter is denoted with $\kappa 3$ and the statistical security parameter with $\sigma$. In practice, one can choose $\kappa=40$ and $\sigma=80$.

We work in the semi-honest model where participants are assumed to be honest-but-curious (details later in \$3). Our improved protocols can be proven in this model based on existing proofs for the basic building blocks from which they are composed. We further note that efficient garbled circuits of [21] (and thus our work) requires the use of random oracles. We could also use correlationrobust hash functions [19, resulting in slightly more expensive computation of garbled circuits 31. (see below).

\footnotetext{
${ }^{3}$ The probability that the protocol computes a wrong result (e.g., caused by an over-
} flow) is bounded by $2^{-\kappa}$. 


\subsection{Cryptographic Tools}

Homomorphic Encryption (HE). We use a semantically secure additively homomorphic public-key encryption scheme. In an additively homomorphic cryptosystem, given encryptions $\llbracket a \rrbracket$ and $\llbracket b \rrbracket$, an encryption $\llbracket a+b \rrbracket$ can be computed as $\llbracket a+b \rrbracket=\llbracket a \rrbracket \llbracket b$, where all operations are performed in the corresponding plaintext or ciphertext structure. From this property follows, that multiplication of an encryption $\llbracket a \rrbracket$ with a constant $c$ can be computed efficiently as $\llbracket c \cdot a \rrbracket=\llbracket a \rrbracket^{c}$ (e.g., with the square-and-multiply method).

As instantiation we use the Paillier cryptosystem [29]11] which has plaintext space $\mathbb{Z}_{N}$ and ciphertext space $\mathbb{Z}_{N^{2}}^{*}$, where $N$ is a $T$-bit RSA modulus. This scheme is semantically secure under the decisional composite residuosity assumption (DCRA). For details on the encryption and decryption function we refer to [11]. The protocol for privacy-preserving face recognition proposed in 12 additionally uses the additively homomorphic cryptosystem of Damgård, Geisler and Krøigård (DGK) which reduces the ciphertext space to $\mathbb{Z}_{N}^{*} 910$.

Oblivious Transfer (OT). For our construction we use parallel 1-out-of-2 Oblivious Transfer for $m$ bitstrings of bitlength $\ell$, denoted as $\mathrm{OT}_{\ell}^{m}$. It is a twoparty protocol where the server $\mathcal{S}$ inputs $m$ pairs of $\ell$-bit strings $S_{i}=\left\langle s_{i}^{0}, s_{i}^{1}\right\rangle$ for $i=1, . ., m$ with $s_{i}^{0}, s_{i}^{1} \in\{0,1\}^{\ell}$. Client $\mathcal{C}$ inputs $m$ choice bits $b_{i} \in\{0,1\}$. At the end of the protocol, $\mathcal{C}$ learns $s_{i}^{b_{i}}$, but nothing about $s_{i}^{1-b_{i}}$ whereas $\mathcal{S}$ learns nothing about $b_{i}$. We use $\mathrm{OT}_{\ell}^{m}$ as a black-box primitive in our constructions. It can be instantiated efficiently with different protocols 25|1|23|19]. It is possible to pre-compute all OTs in a setup phase while the online phase consists of 2 messages with $\Theta(2 m t)$ bits. Additionally, the number of public-key operations in the setup phase can be reduced to be constant with the extensions of [19].

Garbled Circuit (GC). Yao's Garbled Circuit approach [35122], is the most efficient method for secure evaluation of a boolean circuit $C$. We summarize its ideas in the following. First, server $\mathcal{S}$ creates a garbled circuit $\widetilde{C}$ with algorithm CreateGC: for each wire $W_{i}$ of the circuit, he randomly chooses a complementary garbled value $\widehat{w}_{i}=\left\langle\widetilde{w}_{i}^{0}, \widetilde{w}_{i}^{1}\right\rangle$ consisting of two secrets, $\widetilde{w}_{i}^{0}$ and $\widetilde{w}_{i}^{1}$, where $\widetilde{w}_{i}^{j}$ is the garbled value of $W_{i}$ 's value $j$. (Note: $\widetilde{w}_{i}^{j}$ does not reveal $j$.) Further, for each gate $G_{i}, \mathcal{S}$ creates and sends to client $\mathcal{C}$ a garbled table $\widetilde{T}_{i}$ with the following property: given a set of garbled values of $G_{i}$ 's inputs, $\widetilde{T}_{i}$ allows to recover the garbled value of the corresponding $G_{i}$ 's output, and nothing else. Then garbled values corresponding to $\mathcal{C}$ 's inputs $x_{j}$ are (obliviously) transferred to $\mathcal{C}$ with a parallel oblivious transfer protocol OT (see below): $\mathcal{S}$ inputs complementary garbled values $\widetilde{W}_{j}$ into the protocol; $\mathcal{C}$ inputs $x_{j}$ and obtains $\widetilde{w}_{j}^{x_{j}}$ as outputs. Now, $\mathcal{C}$ can evaluate the garbled circuit $\widetilde{C}$ with algorithm EvalGC to obtain the garbled output simply by evaluating the garbled circuit gate by gate, using the garbled tables $\widetilde{T}_{i}$. Finally, $\mathcal{C}$ determines the plain values corresponding to the obtained garbled output values using an output translation table received by $\mathcal{S}$. Correctness of GC follows from method of construction of garbled tables $\widetilde{T}_{i}$. 
Implementation Details. For most efficient implementation of the garbled circuit we use several extensions of Yao's garbled circuit methodology as summarized in 31: the "free XOR" trick of 21] allows "free" evaluation of XOR gates (no communication and negligible computation); for each non-XOR gate (e.g., AND, $\mathrm{OR}, . .$.$) we use garbled row reduction [2631] which allows to omit the first entry$ of the garbled tables, i.e., for each non-XOR gate with 2 inputs a garbled table of $\Theta(3 t)$ bits is transferred; point-and-permute 24 allows fast GC evaluation, i.e., evaluation of a 2 input non-XOR gate requires in the random oracle model one invocation of a suitably chosen cryptographic hash function such as SHA-256. In the standard model, two invocations are needed [31].

Efficient Circuit Constructions. We use the following efficient circuit building

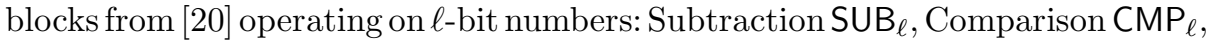
and Multiplexer MUX $_{\ell}$ circuits of size $\ell$ non-XOR gates. Circuits can be automatically generated from a high-level description with the compiler of [30].

\subsection{Face Recognition Using Eigenfaces}

A well-known algorithms for face recognition is the so-called Eigenfaces algorithm introduced in 3433. This algorithm achieves reasonable classification rates of approximately $96 \%$ [12] and is simple enough to be implemented as privacy-preserving protocol (cf. 93). The Eigenfaces algorithm transforms face images into their characteristic feature vectors in a low-dimensional vector space (face space), whose basis consists of Eigenfaces. The Eigenfaces are determined through Principal Component Analysis (PCA) from a set of training images; every face is represented as a vector in the face space by projecting the face image onto the subspace spanned by the Eigenfaces. Recognition is done by first projecting the face image into the face space and afterwards locating the closest feature vector. For details on the enrollment process we refer to [12] and original papers on Eigenfaces 34/33. In the following we briefly summarize the recognition process of the Eigenfaces algorithm. A pseudocode description and the naming conventions and sizes of parameters are given in Appendix

Inputs and Outputs: The algorithm obtains as input the query face image $\Gamma$ represented as a pixel image with $N$ pixels. Additionally, the algorithm obtains the parameters determined in the enrollment phase as inputs: the average face $\Psi$ which is the mean of all training images, the Eigenfaces $u_{1}, . ., u_{K}$ which span the $K$-dimensional face space, the projected faces $\Omega_{1}, . ., \Omega_{M}$ being the projections of the $M$ faces in the database into the face space, and the threshold value $\tau$. The output $r$ of the recognition algorithm is the index of that face in the database which is closest to the query face $\Gamma$ or the special symbol $\perp$ if no match was found, i.e., all faces have a larger distance than the threshold $\tau$.

Recognition Algorithm: The recognition algorithm consists of three phases:

1. Projection: First, the average face $\Psi$ is subtracted from the face $\Gamma$ and the result is projected into the $K$-dimensional face space using the Eigenfaces $u_{1}, . ., u_{K}$. The result is the projected $K$-dimensional face $\bar{\Omega}$. 
2. Distance: Now, the square of the Euclidean distance $D_{i}$ between the projected $K$-dimensional face $\bar{\Omega}$ and all projected $K$-dimensional faces in the database $\Omega_{i}, i=1, . ., M$, is computed.

3. Minimum: Finally, the minimum distance $D_{\min }$ is selected. If $D_{\min }$ is smaller than threshold $\tau$, the index of the minimum value, i.e., the identifier $i_{\min }$ of the match found, is returned to $\mathcal{C}$ as result $r=i_{\text {min }}$. Otherwise, the image was not found and the special symbol $r=\perp$ is returned.

\section{Privacy-Preserving Face Recognition}

Privacy-Preserving Face Recognition allows a client to obliviously detect if the image of a face is contained in a database of faces held by a server. This can be achieved by securely evaluating a face recognition algorithm within a cryptographic protocol. In the following we concentrate on the Eigenface algorithm described in $\$ 2.3$ which was also used in [12. Our techniques can be extended to implement different recognition algorithms as discussed in the full version [32].

\subsection{Privacy-Preserving Face Recognition Using Eigenfaces}

The inputs and outputs of the Eigenfaces algorithm are distributed between client $\mathcal{C}$ and server $\mathcal{S}$ as shown in Fig. 1(a). Both parties want to hide their inputs from the other party during the protocol run, i.e., $\mathcal{C}$ does not want to reveal for which face she is searching while $\mathcal{S}$ does not want to reveal the faces in his database or the details of the applied transformation into the face space (including Eigenfaces which might reveal critical information about faces in DB).

In the semi-honest model we are working in, parties are assumed to follow the protocol but try to learn additional information from the protocol trace beyond what can be derived from the inputs and outputs of the algorithm when used as a black-box. In particular this requires that all internal results of the Eigenfaces algorithm, including the values passed between the different phases $\bar{\Omega}$ and $D_{1}, . ., D_{M}$, are "hidden" from both parties. For practical applications it is sufficient to assume that both parties are computationally bounded, i.e., no polynomial-time adversary can derive information from "hidden" values.

For implementing the privacy-preserving Eigenfaces algorithm and "hiding" the intermediate values, different techniques can be used as listed in Fig. 1(b).

To the best of our knowledge, the only previous work on privacy-preserving face recognition [12] uses homomorphic encryption (HE) to implement the Eigenfaces algorithm in a privacy-preserving way, i.e., computations are performed on homomorphically encrypted data and the intermediate values are homomorphically encrypted (denoted as $\llbracket \cdot \rrbracket)$. We summarize this protocol in $\$ 3.2$.

Our Hybrid protocol presented in $\$ 4.1$ substantially improves the efficiency of this protocol by implementing the Projection and Distance phase using homomorphic encryption and the Minimum phase with a garbled circuit. An alternative protocol which is entirely based on garbled circuits and hides intermediate val-

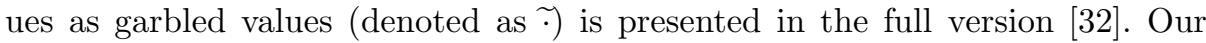
improvements over previous work are summarized in $\$ 5$. 
Client $\mathcal{C}$

Server $\mathcal{S}$

face $\Gamma$

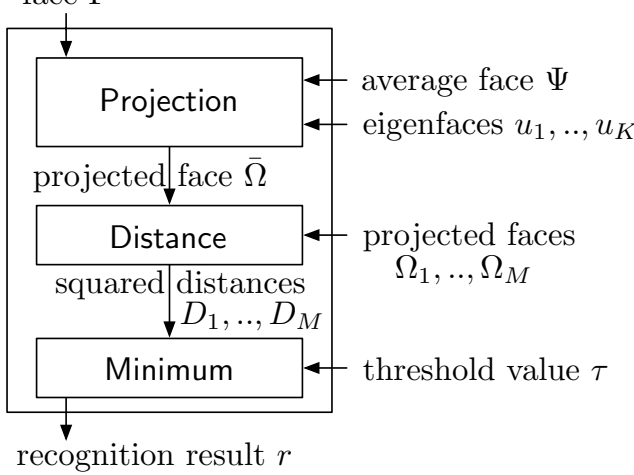

(a) Protocol Structure

\begin{tabular}{|c||c||c|c|}
\hline \multicolumn{1}{|c||}{} & $\llbracket 12$ & \multicolumn{2}{c|}{ This Work } \\
\hline Protocol & HE & Hybrid & GC \\
& $(\Phi 3.2)$ & $(\$ 4.1)$ & {$[32$} \\
\hline \hline Projection & HE & HE & GC \\
$\downarrow$ & $\llbracket \bar{\Omega} \rrbracket$ & $\llbracket \bar{\Omega} \rrbracket$ & $\widetilde{\bar{\Omega}}$ \\
Distance & $\mathrm{HE}$ & $\mathrm{HE}$ & $\mathrm{GC}$ \\
$\downarrow$ & $\llbracket D_{i} \rrbracket_{i=1}^{M}$ & $\llbracket D_{i} \rrbracket_{i=1}^{M}$ & $\left(\widetilde{D}_{i}\right)_{i=1}^{M}$ \\
Minimum & $\mathrm{HE}$ & $\mathrm{GC}$ & $\mathrm{GC}$ \\
\hline
\end{tabular}

(b) Protocols and Applied Techniques

Fig. 1. Privacy-Preserving Face Recognition using Eigenfaces

\subsection{Previous Work: Privacy-Preserving Face Recognition Using HE}

In 12, the authors describe describe a protocol for privacy-preserving face recognition which implements the Eigenfaces recognition algorithm of $\$ 2.3$ on homomorphically encrypted data. Their protocol is secure in the semi-honest model, i.e., players are honest-but-curious [12, Appendix A].

Projection. First, $\mathcal{C}$ and $\mathcal{S}$ jointly compute the projection of the face image $\Gamma$ into the eigenspace spanned by the Eigenfaces $u_{1}, . ., u_{K}$ as follows: $\mathcal{C}$ generates a secret/public key pair of a homomorphic encryption scheme (cf. \2.2) and encrypts the face $\Gamma$ as $\llbracket \Gamma \rrbracket=\left(\llbracket \Gamma_{1} \rrbracket, . ., \llbracket \Gamma_{N} \rrbracket\right) . \mathcal{C}$ sends the encrypted face $\llbracket \Gamma \rrbracket$ along with the public key to $\mathcal{S}$. Using the homomorphic properties, $\mathcal{S}$ projects the encrypted face into the low-dimensional face space and obtains the encryption of the projected face $\llbracket \bar{\Omega} \rrbracket=\left(\llbracket \bar{\omega}_{1} \rrbracket, . ., \llbracket \bar{\omega}_{K} \rrbracket\right)$ by computing for $i=1, . ., K: \llbracket \bar{\omega}_{i} \rrbracket=$ $\llbracket-\sum_{j=1}^{N} u_{i, j} \Psi_{j} \rrbracket \cdot \prod_{j=1}^{N} \llbracket \Gamma_{j} \rrbracket^{u_{i, j}}$. The first factor can already be computed in the pre-computation phase.

Distance. After Projection, $\mathcal{C}$ and $\mathcal{S}$ jointly compute the encryption of the Euclidean distances between the projected face $\llbracket \bar{\Omega} \rrbracket$ and all projected faces $\Omega_{1}, . ., \Omega_{M}$ in the database held by $\mathcal{S}$. This is done by computing for $i=1, \ldots, M$ : $\llbracket D_{i} \rrbracket=$ $\llbracket|| \Omega_{i}-\bar{\Omega} \|^{2} \rrbracket=\llbracket S_{1, i} \rrbracket \cdot \llbracket S_{2, i} \rrbracket \cdot \llbracket S_{3} \rrbracket$, where $\llbracket S_{1, i} \rrbracket=\llbracket \sum_{j=1}^{K} \omega_{i, j}^{2} \rrbracket=\prod_{j=1}^{K} \llbracket \omega_{i, j}^{2} \rrbracket$ and $\llbracket S_{2, i} \rrbracket=\llbracket \sum_{j=1}^{K}\left(-2 \omega_{i, j} \bar{\omega}_{j}\right) \rrbracket=\prod_{j=1}^{K} \llbracket \bar{\omega}_{j} \rrbracket^{-2 \omega_{i, j}}$ can be computed by $\mathcal{S}$ from $\llbracket \bar{\Omega} \rrbracket$ without interaction with $\mathcal{C}$. To obtain $\llbracket S_{3} \rrbracket=\llbracket \sum_{j=1}^{K} \bar{\omega}_{j}^{2} \rrbracket$ from $\llbracket \bar{\Omega} \rrbracket$, the following protocol is suggested in [12]: For $j=1, . ., K: \mathcal{S}$ chooses $r_{j} \in R \mathbb{Z}_{n}$, computes $\llbracket x_{j} \rrbracket=\llbracket \bar{\omega}_{j}+r_{j} \rrbracket=\llbracket \bar{\omega}_{j} \rrbracket \cdot \llbracket r_{j} \rrbracket$ and sends $\llbracket x_{j} \rrbracket$ to $\mathcal{C}$. $\mathcal{C}$ decrypts $\llbracket x_{j} \rrbracket$, computes $\llbracket S_{3}^{\prime} \rrbracket=\llbracket \sum_{j=1}^{K} x_{j}^{2} \rrbracket$, and sends $\llbracket S_{3}^{\prime} \rrbracket$ to $\mathcal{S}$. $\mathcal{S}$ finally computes $\llbracket S_{3} \rrbracket=$ $\llbracket S_{3}^{\prime} \rrbracket \cdot \llbracket-\sum_{j=1}^{K} r_{j}^{2} \rrbracket \cdot \prod_{j=1}^{K} \llbracket \bar{\omega}_{j} \rrbracket^{-2 r_{j}}$. 
Minimum. As last step, $\mathcal{C}$ and $\mathcal{S}$ jointly compute the minimum value $D$ from $\llbracket D_{1} \rrbracket, . ., \llbracket D_{M} \rrbracket$ and its index Id. If the minimum value $D$ is smaller than the threshold value $\tau$ known by $\mathcal{S}$, then $\mathcal{C}$ obtains the result Id. To achieve this, 12 suggests the following protocol: Choose the minimum value and index from the list of encrypted value and id pairs $\left(\llbracket D_{0}=\tau \rrbracket, \llbracket \operatorname{ld}_{0}=\perp \rrbracket\right),\left(\llbracket D_{i} \rrbracket, \llbracket \operatorname{Id}_{i} \rrbracket\right)_{i=1}^{M}$. For this, they apply a straight-forward recursive algorithm for minimum selection based on a sub-protocol which compares two encrypted distances and returns a re-randomized encryption of the minimum and its index to $\mathcal{S}$. For this subprotocol, an optimized version of the homomorphic encryption-based comparison protocol of Damgård, Geisler and Krøigaard (DGK) 910] is used.

Complexity of Minimum protocol (cf. Table [1). The Minimum protocol of [12] requires a logarithmic number of $6\left\lceil\log _{2}(M+1)\right\rceil+1$ moves. Overall, $8 M$ Paillier ciphertexts and $2 \ell^{\prime} M$ DGK ciphertexts are sent in the online phase, where $\ell^{\prime}=50$ is the length of the squared distances $D_{1}, . ., D_{M}$ among which the minimum is selected (cf. Table 3 in Appendix 4 ). This results in a communication complexity of $\left(16+2 \ell^{\prime}\right) M T$ bits. The asymptotic online computation complexity is dominated by approximately $2 M$ Paillier decryptions and $\ell^{\prime} M$ DGK decryptions for $\mathcal{C}$ and the same number of exponentiations for $\mathcal{S}$.

\section{Our Protocols for Privacy-Preserving Face Recognition}

In the following we present our Hybrid protocol which improves over the protocol of [12] (cf. 33.2) and is better suited for larger database sizes. An alternative protocol based on garbled circuits only is given in the full version [32].

\subsection{Privacy-Preserving Face Recognition Using Hybrid of HE + GC}

Our hybrid protocol for privacy-preserving face recognition improves over the protocol in [12] by replacing the Minimum protocol with a more efficient protocol based on garbled circuits. Additionally, the Distance protocol proposed in [12] can be slightly improved by packing together the messages sent from server $\mathcal{S}$ to client $\mathcal{C}$ into a single ciphertext as detailed in the full version [32. We concentrate on the core improvements of the Minimum protocol in the following.

\section{Hybrid Minimum Protocol}

The most efficient protocols for secure comparison in the setting with two computationally bounded parties is still based on Yao's garbled circuit (GC) approach $[35|26| 20]$ as briefly explained in $\$ 2.2$. This also includes the natural generalization to selecting the minimum value and index of multiple values. As shown in [20, these GC based protocols clearly outperform comparison protocols based on homomorphic encryption [13/614/9/10]. In the following we show how the protocols of [20] can be adopted to yield a highly efficient, constant round Minimum protocol for our Hybrid privacy-preserving face recognition protocol. 
Overview. The high-level structure of our improved Minimum protocol is shown in Fig. 3(a) in Appendix $₫ \mathrm{~B}$ and consists of several building-blocks: the subprotocol ParallelConvert converts the homomorphically encrypted distances held by server $\mathcal{S}, \llbracket D_{1} \rrbracket, . ., \llbracket D_{M} \rrbracket$, into their corresponding garbled values $\widetilde{D}_{1}, . ., \widetilde{D}_{M}$ output to client $\mathcal{C}$ (details below). These garbled values are used to evaluate a garbled circuit $\widetilde{C}_{\text {Minimum }}$ which computes the Minimum phase of Algorithm 1 in Appendix $\$ \mathrm{~A}$ (details on how the underlying circuit $C_{\text {Minimum }}$ is constructed below). The garbled circuit $\widetilde{C}_{\text {Minimum }}$ can be created already in the setup phase using algorithm CreateGC and sent to $\mathcal{C}$ before the online phase starts. The garbled values $\widetilde{\tau}$ which correspond to server's threshold value $\tau$ are selected by $\mathcal{S}$ (Select) and transferred to $\mathcal{C}$ as well (either in the setup phase or in the online phase depending on how often the database changes). Finally, $\mathcal{C}$ evaluates $\widetilde{C}_{\text {Minimum }}$ on the garbled values $\widetilde{\tau}, \widetilde{D}_{1}, \ldots, \widetilde{D}_{M}$ and obtains the correct output $r$.

ParallelConvert protocol. An efficient ParallelConvert protocol is given in [20] which we summarize in the following (see [20 and 4] for a detailed description): $\mathcal{S}$ blinds the homomorphically encrypted $\ell^{\prime}$-bit values $\llbracket D_{i} \rrbracket, i=1, . ., M$ with a randomly chosen additive $T$-bit mask $R_{i} \in_{R} \mathbb{Z}_{n}$ and sends the blinded values $\llbracket D_{i}+R_{i} \rrbracket$ to $\mathcal{C}$ who can decrypt. Then, $\mathcal{C}$ and $\mathcal{S}$ jointly run a garbled circuit protocol in order to obliviously take off the mask $R_{i}$ with a subtraction circuit. For improved efficiency, multiple values $\llbracket D_{i} \rrbracket$ can be packed together into a single ciphertext before blinding. To avoid an overflow when adding the $T$-bit random mask, the most significant $\kappa$ bits are left as correctness margin, where $\kappa$ is a statistical correctness parameter (e.g., $\kappa=40$ ). This allows to pack $M^{\prime}=\left\lfloor\frac{T-\kappa}{\ell^{\prime}}\right\rfloor$ values into one ciphertext resulting in $m=\left\lceil\frac{M}{M^{\prime}}\right\rceil$ packed Paillier ciphertexts for the $M$ values. The ParallelConvert protocol consists of 3 moves.

Circuit $C_{\text {Minimum }}$ which computes the required functionality of the Minimum protocol is shown in Fig. $3(\mathrm{~b})$ in Appendix $\mathrm{B}$. First, the minimum value $D_{\min }=$ $\min \left(D_{1}, . ., D_{M}\right)$ and the corresponding index $i_{\min } \in\{1, . ., M\}$ are computed with the MIN circuit. The MIN circuit is similar to the circuit evaluated in a first-price auction where the highest bid and the index of the highest bidder is selected [26. An efficient construction of this circuit has size $|\mathrm{MIN}| \sim 2 \ell^{\prime} M$ non-XOR gates [20]. Afterwards, the minimum value $D_{\min }$ is compared with the threshold value $\tau$ using a comparison circuit CMP. The output $c$ of the CMP circuit is 1 if $D_{\min } \leq \tau$ and 0 otherwise. Depending on $c$, the multiplexer MUX chooses either the minimum index $i_{\min }$ if $c=1$ as output or the special symbol $\perp$ otherwise (e.g., $\perp=0$ ). The circuit has size $\left|C_{\text {Minimum }}\right| \sim 2 \ell^{\prime} M$ non-XOR gates.

Complexity. The complexity of our improved Minimum protocol and the one proposed in [12] is given in Table 1. For the computation complexity the table contains only the dominant costs: the number of Paillier and Damgård-GeislerKrøigård (DGK) decryptions (Dec) and exponentiations (Exp) as well as the number of evaluations of a cryptographic hash function (Hash). 
Table 1. Complexity of Minimum Protocols with Parameters $M$ : \# faces in database, $\ell^{\prime}$ : bitlength of values $D_{1}, . ., D_{M}, t$ : symmetric security parameter, $T$ : asymmetric security parameter, $\kappa$ : statistical correctness parameter, $m \sim \frac{\ell^{\prime}}{T-\kappa} M$

\begin{tabular}{|c|c|c|}
\hline & HE $\$ 3.212$ & Hybrid $\$ 4.1$ \\
\hline Round Complexity & $6\lceil\log (M+1)\rceil+1$ moves & 3 moves \\
\hline \multicolumn{3}{|c|}{ Asymptotic Communication Complexity [bits] } \\
\hline online & $\left(2 \ell^{\prime}+16\right) M T$ & $2 \ell^{\prime} M t+2 m T$ \\
\hline offline & & $\mathrm{OT}_{t}^{\ell^{\prime} M}+9 \ell^{\prime} M t$ \\
\hline \multicolumn{3}{|c|}{ Asymptotic Computation Complexity } \\
\hline $\mathcal{C}$ online & $\approx 2 M$ Dec $_{\text {Paillier }}+\ell^{\prime} M \operatorname{Dec}_{\text {DGK }}$ & $m$ Dec $_{\text {Paillier }}+3 \ell^{\prime} M \mathrm{Ha}$ \\
\hline $\mathcal{S}$ online & $\approx 2 M \operatorname{Exp}_{\text {Paillier }}+\ell^{\prime} M \operatorname{Exp}_{\mathrm{DGK}}$ & $m \operatorname{Exp}_{\text {Paillier }}$ \\
\hline
\end{tabular}

Our improved Minimum protocol requires a constant number of 3 moves for the ParallelConvert protocol ( $\widetilde{\tau}$ can be sent with the last message). The online communication complexity is determined by the ParallelConvert protocol for converting $M$ values of bitlength $\ell^{\prime}$, i.e., $m$ Paillier ciphertexts and the online part of the $\mathrm{OT}_{t}^{\ell^{\prime} M}$ protocol which is asymptotically $2 \ell^{\prime} M t+2 m T$ bits (cf. 2.2 ). The online computation complexity requires $\mathcal{S}$ to pack the $m$ ciphertexts (corresponds to $m$ exponentiations) and $\mathcal{C}$ to decrypt them. After the OT protocol, $\mathcal{C}$ needs to evaluate a garbled circuit consisting of approximately $3 \ell^{\prime} M$ non-XOR gates $\left(\ell^{\prime} M\right.$ to subtract the random masks in the ParallelConvert protocol and $2 \ell^{\prime} M$ for $C_{\text {Minimum }}$ ) which requires to invoke a cryptographic hash function (e.g., SHA-256) the same number of times. The offline communication consists of the $\mathrm{OT}_{t}^{\ell^{\prime} M}$ protocol and transferring the GC (3t bits per non-XOR gate, cf. $₫ 2.2$ ).

Improvements (cf. Table 1). Most notably, the round complexity of our improved Minimum protocol is independent of the size $M$ of the database.

The online communication complexity of our protocol is smaller by a factor of approximately $T / t$, e.g., $1024 / 80 \approx 13$ for short-term security and 38 for long-term security (see $\$ 5.1$ for details).

The online computation complexity of our protocol is substantially lower, as the number of Paillier operations is reduced by a factor of approximately $2 M / m=2 M^{\prime}=\frac{2(T-\kappa)}{\ell^{\prime}}$, e.g., $\frac{2(1024-40)}{50} \approx 40$ for short-term security and 121 for long-term security. GC evaluation (which requires one invocation of SHA-256 per gate) is computationally less expensive than the modular arithmetics needed for the DGK public-key cryptosystem used in [12] (see \$5.2 for details).

\section{Complexity Improvements}

In the following we compare our improved protocol with the protocol of [12]: communication- and round complexity in $\$ 5.1$ and computation complexity in $\$ 5.2$. We consider different recommended sizes of security parameters for short-, medium-, and long-term security [16] (cf. Appendix §C for parameter sizes). 


\subsection{Round Complexity and Asymptotic Communication Complexity}

HE vs. Hybrid (Table 2). Our Hybrid protocol substantially improves the performance of the HE protocol proposed in [12]: the round complexity is reduced from logarithmic in the size of the database $M$ down to a small constant of 6 moves. The online communication complexity of the Minimum protocol (\$4.1) is reduced to only $6.6 \%$ of the previous solution for short-term security. For medium- and long-term security the savings are even better. Our improvements of the Distance protocol (in full version [32]) down to $23 \%$ for short-term security are negligible w.r.t. the overall communication complexity as it has small communication complexity (few KBytes) independent of the database size $M$.

Table 2. Round- and Communication Complexity - HE vs. Hybrid. $M$ : size of DB

\begin{tabular}{|c|c|c|c|c|c|c|}
\hline Protocol & \multicolumn{3}{|c|}{ HE $₫ 3.212$} & \multicolumn{3}{|c|}{ Hybrid 4.1 (Improvement) } \\
\hline Round Complexity [moves] & $6\lceil\log$ & $M+1)$ & $7+4$ & $(\mathcal{C}$ & $(\log M) \rightarrow$ & $\mathcal{O}(1))$ \\
\hline Security Level & Short & Medium & Long & Short & Medi & Long \\
\hline \multicolumn{7}{|c|}{ Asymptotic Communication Complexity (online) } \\
\hline tion $[\mathrm{MB}]$ & 2.5 & 5.0 & 7.5 & 2.5 & 5.0 & 7.5 \\
\hline $\mathrm{e}[\mathrm{kB}]$ & 3.2 & 6.5 & 9.8 & $0.75(23 \%)$ & $1.0(15 \%)$ & $1.5(15 \%)$ \\
\hline Minimum $[\mathrm{kB}$ per face in $\mathrm{DB}]$ & 15 & 29 & 44 & $0.99(6.6 \%)$ & $1.4(4.8 \%)$ & $1.6(3.6 \%)$ \\
\hline
\end{tabular}

\subsection{Online Computation Complexity}

Hybrid protocol (\$4.1). We have implemented the Hybrid protocol for privacy-preserving face recognition described in 4.1 in Python to quantify its online computation complexity. Although interpreted Python code runs substantially slower than compiled code we chose it for platform independence. We perform performance measurements on two standard PCs (AMD Athlon64 X2 $5000+(2.6 \mathrm{GHz}), 2$ Cores, 4 GB Memory running on Gentoo Linux x86_64) communicating via TCP/IP6 over a Gigabit Ethernet connection. Both machines were clocked to $2.4 \mathrm{GHz}$ via $\mathrm{CPU}$ frequency scaling to make the performance comparable to [12. The implementation is running in the cPython-2.6 interpreter and uses gmpy module (version 1.04) to access GNU GMP library (version 4.3.1).

In comparison, the protocol in [12 was implemented in $\mathrm{C}++$ using the GNU GMP library (version 4.2.4) and executed on a single PC (2.4 GHz AMD Opteron with dual-core processor and 4 GB RAM under Linux) as two threads. This implementation neglects latencies of communication stack and network which could result in non-negligible slow-downs due to their logarithmic round complexity.

Although our implementation is closer to a real-world setting and uses a substantially slower programming language, it still outperforms that of [12 especially for larger database sizes due to our algorithmic protocol improvements of the Minimum protocol as shown in Fig. 2(a), Surprisingly, our implementation is about $30 \%$ faster than the $\mathrm{C}++$ implementation of [12] even in the homomorphic encryption-based parts of the protocol (Projection and Distance). Presumably this is due to faster multiplication in GMP version 4.3. 


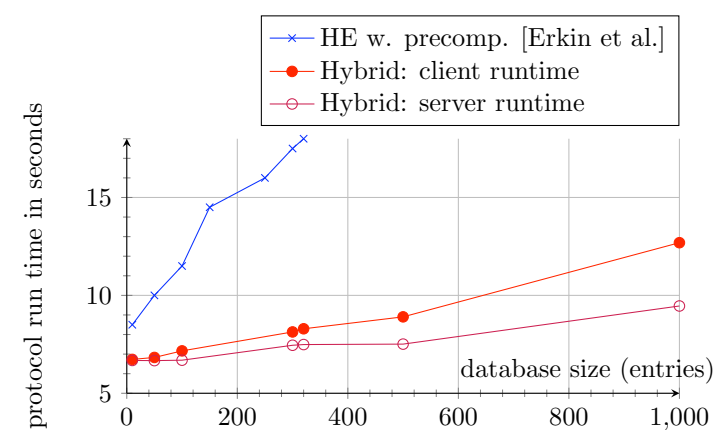

\begin{tabular}{|l||r|r|r|}
\hline \multicolumn{1}{|c||}{} & \multicolumn{3}{c|}{ Security Level } \\
\hline \hline Client & Short & Medium & Long \\
\hline Projection & 0.49 & 0.60 & 0.72 \\
Distance & 6.08 & 16.87 & 31.73 \\
Minimum & 1.86 & 2.71 & 4.49 \\
\hline Sum & 8.43 & 20.18 & 36.95 \\
\hline \hline Server & Short & Medium & Long \\
\hline Projection & 6.58 & 17.43 & 32.37 \\
Distance & 0.47 & 1.52 & 3.03 \\
Minimum & 0.06 & 0.21 & 0.54 \\
\hline Sum & 7.11 & 19.15 & 35.94 \\
\hline
\end{tabular}

(a) HE vs. Hybrid Protocol (Short-Term Security)

(b) Hybrid Protocol for $M=320$

Fig. 2. Comparison of Timing Complexity in [s]

In contrast to the HE-based protocol of [12], our protocol scales well with increasing security level as shown in Fig. 2(b), as symmetric security parameter $t$ increases much slower than its asymmetric equivalent $T$ (cf. Appendix $\mathrm{C}$ ).

Overall, the implementation results confirm that our Hybrid protocol allows privacy-preserving face recognition even for large databases.

\subsection{Conclusion and Future Work}

The methods for constructing efficient protocols for privacy-preserving face recognition presented in this paper can be further improved into various directions.

Algorithmic Improvements for better classification accuracy might be achieved by using different face recognition algorithms. Fisherfaces [5], which determine the projection matrix with Linear Discriminant Analysis (LDA), can be used instead of Eigenfaces. A different distance metric than Euclidean distance could be used, e.g., Hamming distance or Manhattan distance. The Minimum phase could be based on meaning or scoring instead of minimum selection.

Further Protocol Improvements could be achieved with a different homomorphic encryption scheme that allows both, additions and multiplications [7/2 15] to avoid the additional communication round for computing Euclidean Distance.

Further Implementation Improvements can be achieved by exploiting parallelism on multi-core architectures or graphics processing units (GPUs).

Acknowledgements. We thank Wilko Henecka for extending the compiler of [30] to generate the underlying circuits, authors of [12] for detailed information on their protocol, and anonymous reviewers of ICISC 2009 for helpful comments.

\section{References}

1. Aiello, W., Ishai, Y., Reingold, O.: Priced oblivious transfer: How to sell digital goods. In: Pfitzmann, B. (ed.) EUROCRYPT 2001. LNCS, vol. 2045, pp. 119-135. Springer, Heidelberg (2001) 
2. Armknecht, F., Sadeghi, A.-R.: A new approach for algebraically homomorphic encryption. Cryptology ePrint Archive, Report 2008/422 (2008), http://eprint.iacr.org/2008/422

3. Avidan, S., Butman, M.: Efficient methods for privacy preserving face detection. In: Advances in Neural Information Processing Systems (NIPS'06), pp. 57-64. MIT Press, Cambridge (2006)

4. Barni, M., Failla, P., Kolesnikov, V., Lazzeretti, R., Sadeghi, A.-R., Schneider, T.: Secure evaluation of private linear branching programs with medical applications. In: Backes, M., Ning, P. (eds.) ESORICS 2009. LNCS, vol. 5789, pp. 424-439. Springer, Heidelberg (2009)

5. Belhumeur, P.N., Hespanha, J.P., Kriegman, D.J.: Eigenfaces vs. Fisherfaces: Recognition using class specific linear projection. IEEE Transactions on Pattern Analysis and Machine Intelligence 19(7), 711-720 (1997)

6. Blake, I.F., Kolesnikov, V.: Strong conditional oblivious transfer and computing on intervals. In: Lee, P.J. (ed.) ASIACRYPT 2004. LNCS, vol. 3329, pp. 515-529. Springer, Heidelberg (2004)

7. Boneh, D., Goh, E.-J., Nissim, K.: Evaluating 2-DNF formulas on ciphertexts. In: Kilian, J. (ed.) TCC 2005. LNCS, vol. 3378, pp. 325-341. Springer, Heidelberg (2005)

8. Bowcott, O.: Interpol wants facial recognition database to catch suspects. Guardian (October 20, 2008), http://www.guardian.co.uk/world/2008/oct/20/interpol-facial-recognition

9. Damgård, I.B., Geisler, M., Krøigård, M.: Efficient and secure comparison for online auctions. In: Pieprzyk, J., Ghodosi, H., Dawson, E. (eds.) ACISP 2007. LNCS, vol. 4586, pp. 416-430. Springer, Heidelberg (2007)

10. Damgård, I., Geisler, M., Krøigård, M.: A correction to efficient and secure comparison for on-line auctions. Cryptology ePrint Archive, Report 2008/321 (2008), http: //eprint.iacr.org/2008/321

11. Damgård, I., Jurik, M.: A generalisation, a simplification and some applications of Paillier's probabilistic public-key system. In: Kim, K.-c. (ed.) PKC 2001. LNCS, vol. 1992, pp. 119-136. Springer, Heidelberg (2001)

12. Erkin, Z., Franz, M., Guajardo, J., Katzenbeisser, S., Lagendijk, I., Toft, T.: Privacy-preserving face recognition. In: Goldberg, I., Atallah, M.J. (eds.) Privacy Enhancing Technologies. LNCS, vol. 5672, pp. 235-253. Springer, Heidelberg (2009)

13. Fischlin, M.: A cost-effective pay-per-multiplication comparison method for millionaires. In: Naccache, D. (ed.) CT-RSA 2001. LNCS, vol. 2020, pp. 457-472. Springer, Heidelberg (2001)

14. Garay, J.A., Schoenmakers, B., Villegas, J.: Practical and secure solutions for integer comparison. In: Okamoto, T., Wang, X. (eds.) PKC 2007. LNCS, vol. 4450, pp. 330-342. Springer, Heidelberg (2007)

15. Gentry, C.: Fully homomorphic encryption using ideal lattices. In: ACM Symposium on Theory of Computing (STOC'09), pp. 169-178. ACM, New York (2009)

16. Giry, D., Quisquater, J.-J.: Cryptographic key length recommendation (March 2009), http://keylength.com

17. Grose, T.: When surveillance cameras talk. Time Magazine (February 11, 2008), http://www.time.com/time/world/article/0, 8599, 1711972,00.html

18. Interational Civil Aviation Organization (ICAO). Machine Readable Travel Documents (MRTD), Doc 9303, Part 1, 5th (edn.) (2003)

19. Ishai, Y., Kilian, J., Nissim, K., Petrank, E.: Extending oblivious transfers efficiently. In: Boneh, D. (ed.) CRYPTO 2003. LNCS, vol. 2729, pp. 145-161. Springer, Heidelberg (2003) 
20. Kolesnikov, V., Sadeghi, A.-R., Schneider, T.: Improved garbled circuit building blocks and applications to auctions and computing minima. In: Cryptology and Network Security (CANS '09). LNCS. Springer, Heidelberg (2009), http://www. springerlink. com/content/175204h13h038581

21. Kolesnikov, V., Schneider, T.: Improved garbled circuit: Free XOR gates and applications. In: Aceto, L., Damgård, I., Goldberg, L.A., Halldórsson, M.M., Ingólfsdóttir, A., Walukiewicz, I. (eds.) ICALP 2008, Part II. LNCS, vol. 5126, pp. 486-498. Springer, Heidelberg (2008)

22. Lindell, Y., Pinkas, B.: A proof of Yao's protocol for secure two-party computation. ECCC Report TR04-063, Electronic Colloquium on Computational Complexity, ECCC (2004)

23. Lipmaa, H.: Verifiable homomorphic oblivious transfer and private equality test. In: Laih, C.-S. (ed.) ASIACRYPT 2003. LNCS, vol. 2894, pp. 416-433. Springer, Heidelberg (2003)

24. Malkhi, D., Nisan, N., Pinkas, B., Sella, Y.: Fairplay a secure two-party computation system. In: USENIX (2004), http://fairplayproject.net

25. Naor, M., Pinkas, B.: Efficient oblivious transfer protocols. In: ACM-SIAM Symposium On Discrete Algorithms (SODA'01), pp. 448-457. Society for Industrial and Applied Mathematics (2001)

26. Naor, M., Pinkas, B., Sumner, R.: Privacy preserving auctions and mechanism design. In: ACM Conference on Electronic Commerce, pp. 129-139 (1999)

27. Naumann, I., Hogben, G.: Privacy features of European eID card specifications. Network Security 2008(8), 9-13 (2008); European Network and Information Security Agency (ENISA)

28. Newton, E.M., Sweeney, L., Malin, B.: Preserving privacy by de-identifying face images. IEEE Transactions on Knowledge and Data Engineering 17(2), 232-243 (2005)

29. Paillier, P.: Public-key cryptosystems based on composite degree residuosity classes. In: Stern, J. (ed.) EUROCRYPT 1999. LNCS, vol. 1592, pp. 223-238. Springer, Heidelberg (1999)

30. Paus, A., Sadeghi, A.-R., Schneider, T.: Practical secure evaluation of semiprivate functions. In: Abdalla, M., Pointcheval, D., Fouque, P.-A., Vergnaud, D. (eds.) ACNS 2009. LNCS, vol. 5536, pp. 89-106. Springer, Heidelberg (2009), http:// www.trust.rub.de/FairplaysPF

31. Pinkas, B., Schneider, T., Smart, N.P., Williams, S.C.: Secure two-party computation is practical. In: Advances in Cryptology ASIACRYPT 2009. LNCS, Springer, Heidelberg (2009), http://www.springerlink. com/content/3p52033869154003

32. Sadeghi, A.-R., Schneider, T., Wehrenberg, I.: Efficient privacy-preserving face recognition. Cryptology ePrint Archive, Report 2009/507 (2009), http://eprint. iacr.org/2009/507

33. Turk, M., Pentland, A.: Eigenfaces for recognition. Journal of Cognitive Neu- roscience 3(1), 71-86 (1991)

34. Turk, M., Pentland, A.: Face recognition using eigenfaces. In: IEEE Computer Vision and Pattern Recognition (CVPR'91), pp. 586-591. IEEE, Los Alamitos (1991)

35. Yao, A.C.: How to generate and exchange secrets. In: IEEE Symposium on Foundations of Computer Science (FOCS'86), pp. 162-167. IEEE, Los Alamitos (1986) 


\section{A Face Recognition Using Eigenfaces: Details}

Algorithm 1 shows the pseudocode description of the Eigenfaces algorithm and Table 3 the naming conventions and sizes of the parameters.

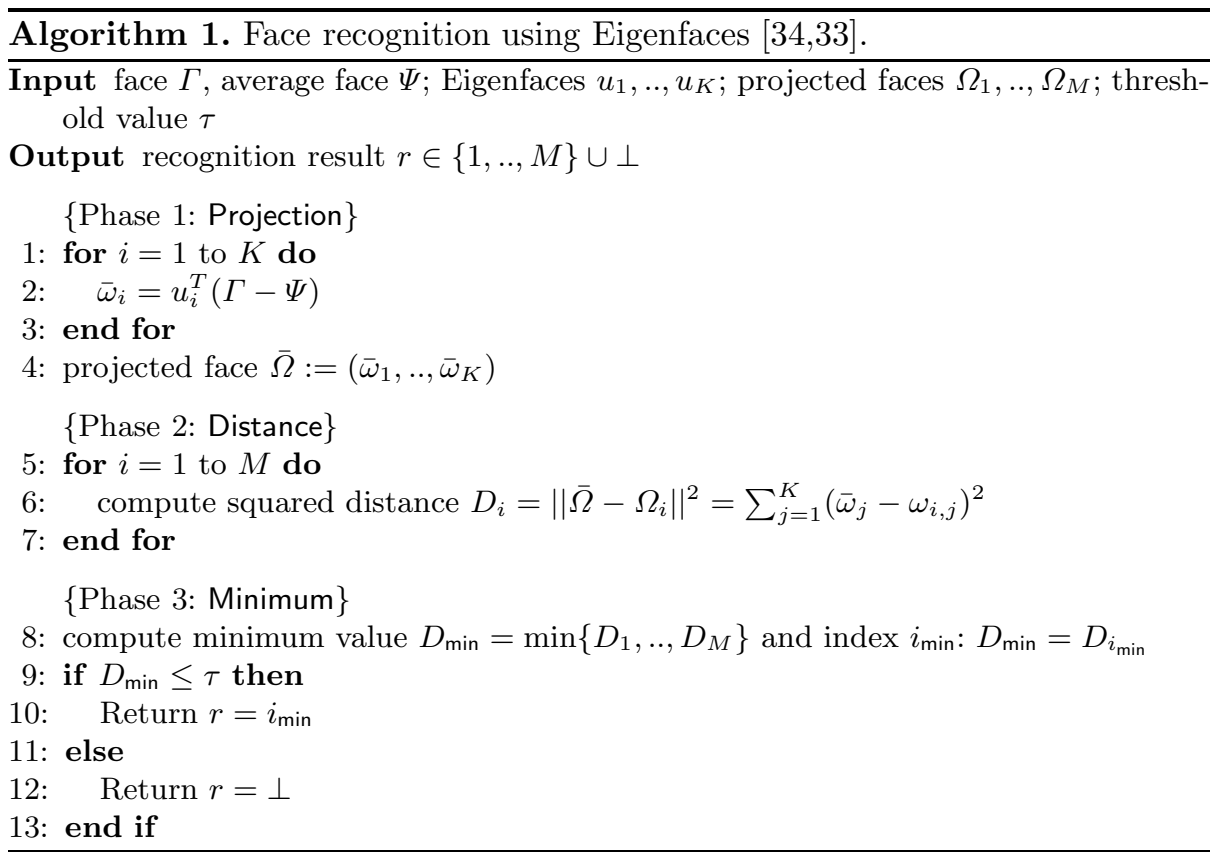

Table 3. Parameters and Sizes for Privacy-Preserving Face Recognition

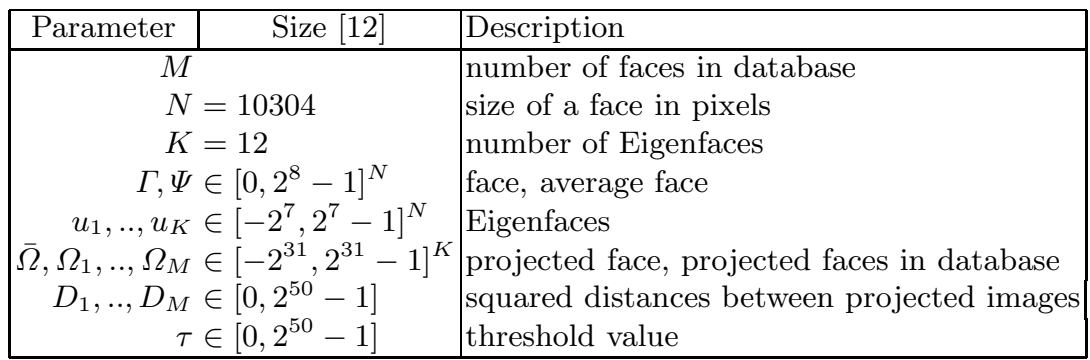




\section{B Improved Minimum Protocol: Details}

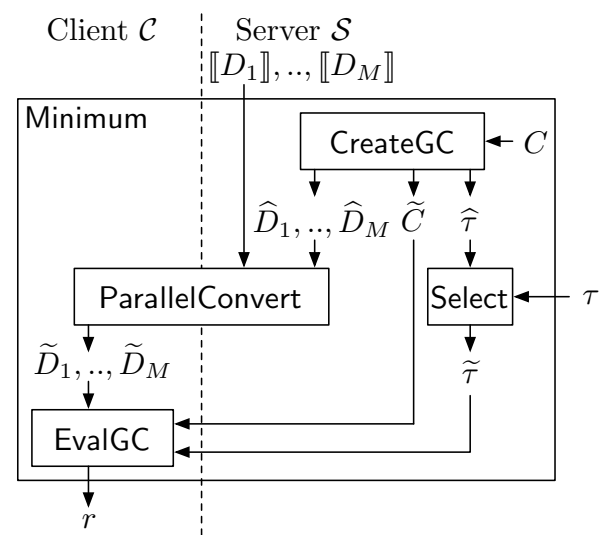

(a) Protocol Structure with $C:=C_{\text {Minimum }}$.

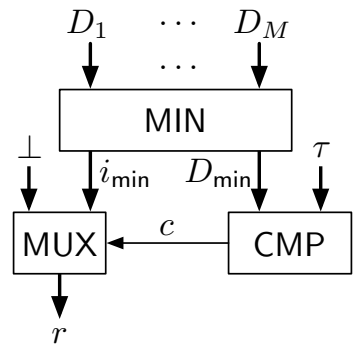

(b) Circuit $C_{\text {Minimum }}$

Fig. 3. Improved Minimum Protocol

\section{Parameter Sizes}

We compare the complexity for different recommended sizes of security parameters - short-term (recommended use up to 2010), medium-term (up to 2030) and long-term security 16. The sizes for the security parameters and corresponding parameter sizes for our Hybrid protocol are summarized in Table 4 we use statistical security parameter $\sigma=80$ and statistical correctness parameter $\kappa=40$. According to Table 3 , the input length for the Distance protocol is $\ell=32$ and for the Minimum protocol (\$4.1) is $\ell^{\prime}=50$.

Table 4. Size of Security Parameters ( $t$ : symmetric security parameter, $T$ : asymmetric security parameter) and Corresponding Parameters for Hybrid Protocol ( $M^{\prime}$ : \# values packed into one ciphertext before blinding)

\begin{tabular}{|l||c|c||c|}
\hline \multicolumn{1}{|c||}{ Security Level } & \multicolumn{2}{c||}{ Security Parameters } & Minimum (\$4.1) \\
& $t$ & $T$ & $M^{\prime}$ \\
\hline Short-Term & 80 & 1024 & 19 \\
Medium-Term & 112 & 2048 & 40 \\
Long -Term & 128 & 3072 & 60 \\
\hline
\end{tabular}

